\title{
Solid Tumours
}

\author{
Ruth Ladenstein, Evgenia Glogova, \\ and Francesco Lanza
}

\subsection{Introduction}

In the absence of randomized prospective trials, the EBMT registry remains an important source to survey indications, outcome and clinical risk factors in patients with solid tumours treated by auto- and allo-HSCT. In early 2018, the EBMT registry includes 57,888 transplant procedures in 42,106 patients, with a slight prevalence in adults compared with children (58\% vs. 42\%). AutoHSCT represents $97 \%$ of the total HSCT, whereas allo-HSCT was used in $3 \%$ of the procedures. Multiple transplants were performed in $1 / 3$ of the cases (Table 92.1). More than 10,000 transplants were performed in the last 5 years (Passweg et al.

\section{R. Ladenstein $(\bowtie)$}

Studies and Statistics for Integrated Research and Projects, St. Anna Children's Hospital, Department of Paediatrics and Children's Cancer Research Institute (CCRI),

Medical University of Vienna,

Vienna, Austria

e-mail: ruth.ladenstein@ccri.at

E. Glogova

Studies and Statistics for Integrated

Research and Projects, St. Anna

Kinderkrebsforschung e.V.,

Vienna, Austria

\section{F. Lanza}

Hematology and HSCT Unit, Department of Onco-Hematology, Hospital S. Maria delle Croci, Ravenna, Italy
Table 92.1 EBMT registry on HSCT for solid tumours (data updated on January 2018)

\begin{tabular}{|c|c|c|}
\hline Solid tumour registry & \multicolumn{2}{|l|}{57,025} \\
\hline Patients & \multicolumn{2}{|l|}{40,593} \\
\hline Adults/pediatric (\%) & \multicolumn{2}{|l|}{$58 / 42$} \\
\hline Male/female (\%) & \multicolumn{2}{|l|}{$48 / 52$} \\
\hline Auto/allo (\%) & \multicolumn{2}{|l|}{$97 / 3$} \\
\hline$N b$ of $H S C T$ & $\begin{array}{l}\text { Auto } \\
(n=55,240)\end{array}$ & Allo $(n=1755)$ \\
\hline First HSCT & 40,412 & 1173 \\
\hline Second HSCT & 10,144 & 440 \\
\hline Third HSCT & 3589 & 100 \\
\hline Fourth HSCT & 651 & 27 \\
\hline$\geq$ Fifth HSCT & 194 & 9 \\
\hline $\begin{array}{l}\text { Median follow up } \\
\text { (year }<2016)\end{array}$ & $2.4(<1$ to 35$)$ & $1.95(<1$ to 31$)$ \\
\hline
\end{tabular}

2018). Figures 92.1 and 92.2 compare activity and indications between adults and children.

\subsection{Solid Tumours in Children and Adolescents}

Ruth Ladenstein and Evgenia Glogova

\subsubsection{Introduction}

The EBMT registry remains an important source to survey indications, outcome and clinical risk factors in children and adolescents with solid tumours treated by high-dose therapy (HDT) and HSCT. 


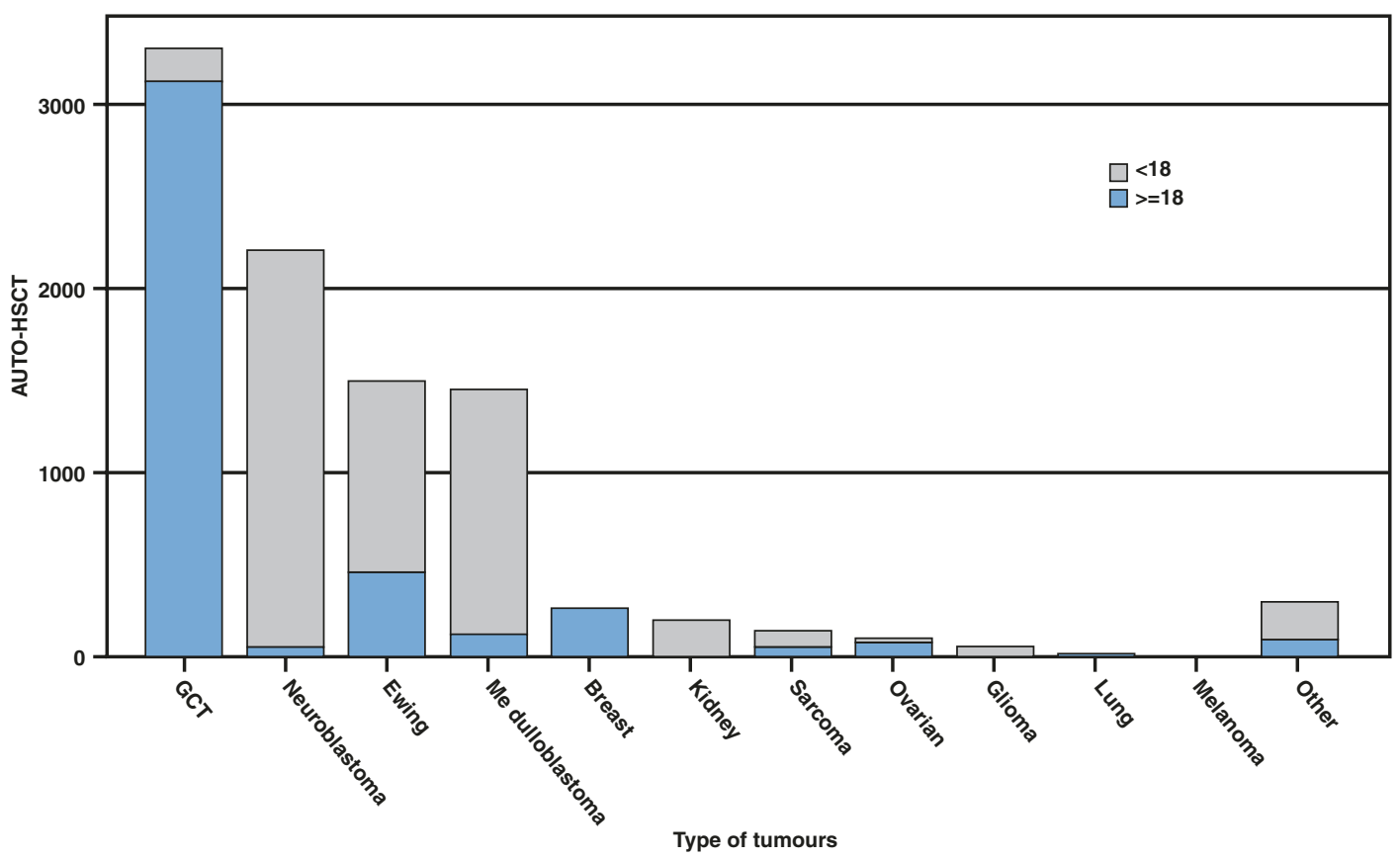

Fig. 92.1 EBMT registry on auto-HSCT for solid tumours in the period 2012-2017 $(n=9519)$. Adults vs. paediatric HSCT is shown (January 2018)

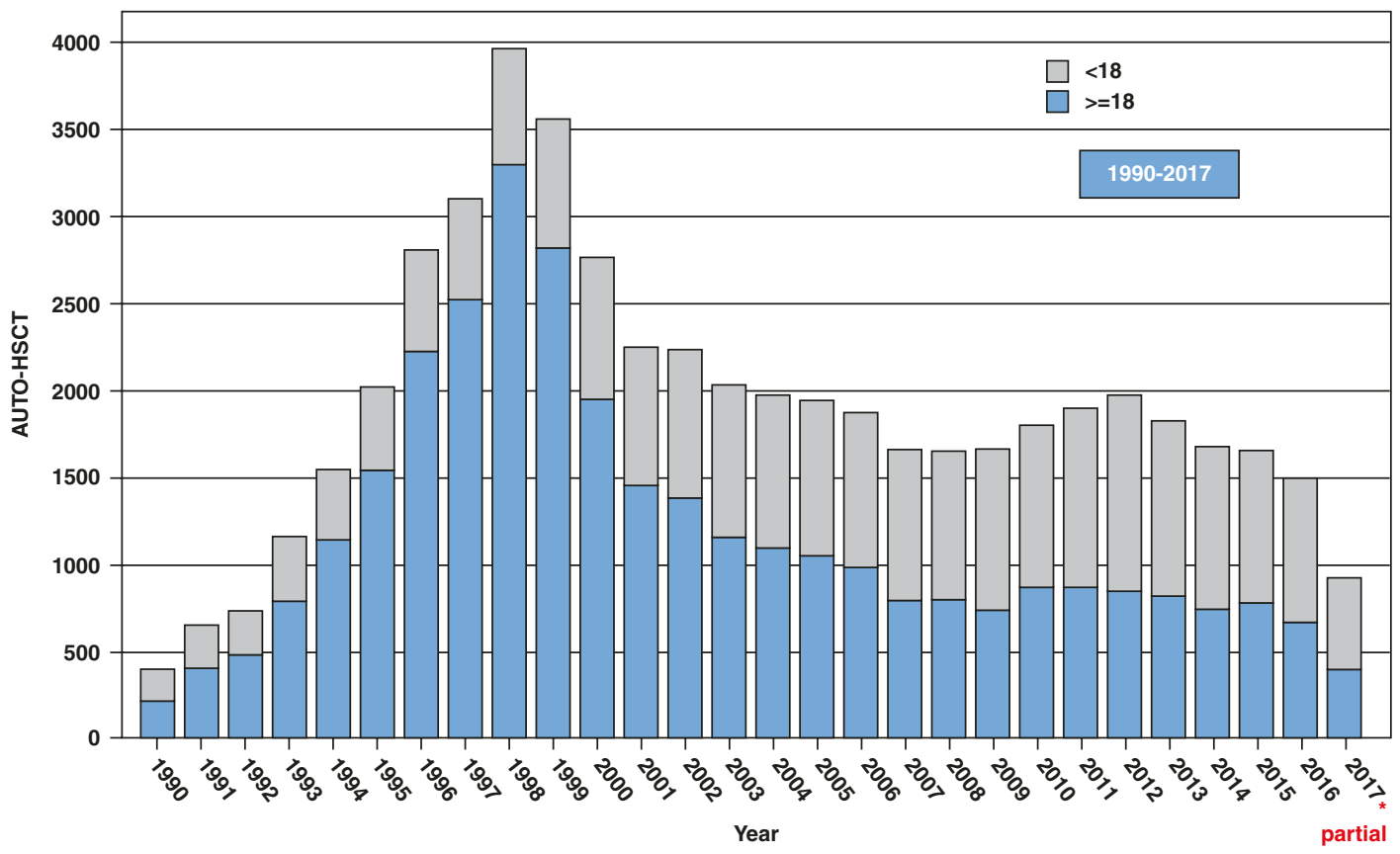

Fig. 92.2 EBMT registry showing numbers of auto-HSCT for solid tumour indication over the years: 1990/2017 in both adults and children 
In view of evolving diagnostic and therapeutic strategies, the definition of high risk needs careful consideration. To date evidence for HDT/ HSCT from prospective randomized trials is still limited to high-risk neuroblastoma and Ewing sarcoma (Matthay et al. 2009; Whelan 2018).

\subsubsection{EBMT Data on HSCT for Solid Tumours in Children and Adolescents}

In 2018 the EBMT database contained information on 17,159 transplants in young people with solid tumours. Their median age is 5.2 years $(0.1-18.0) ; 58 \%$ are male. Auto-HSCT is the most frequent procedure with 14,135 transplants performed, whereas only 446 allo-HSCTs are registered. Data was gathered in 43 countries and reported by 390 centres. The first HSCT was autologous in 16,814 patients and allogeneic in 345 patients. Table 92.2 summarizes the outcomes with auto-HSCT for the most frequent indications for 13,838 with adequate event information in the EBMT database.

\subsubsection{General Lessons from EBMT Data}

The experience of more than 17,000 HSCT procedures in the paediatric age group over 35 years conveys a number of important messages:

Transplant-related mortality. TRM markedly decreased over time and is related with the HDT regimen (i.e. elimination of $\mathrm{TBI}$ ) and most importantly use of peripheral stem cells. TRM rates associated to auto-HSCT dropped to under 5\% after 1992 and is since 2012 only $1 \%$.

Total body irradiation. TBI showed no advantage in any of the solid tumour indications and should thus be avoided in children with solid tumours in view of late effects.

Remission status. First-line high-risk patients perform significantly better than after relapse. Response to induction treatments prior to HDT/HSCT is critical in all indications. A short summary is:

\section{$\mathrm{CR}>\mathrm{VGPR} / \mathrm{PR}>\mathrm{SR} / \mathrm{MR}>\mathrm{NR}(\mathrm{SD})>\mathrm{RR} / \mathrm{UR}$ [CR complete response, $V G P R$ very good partial response, $P R$ partial response ( $>50 \%)$, SR sensitive relapse $=>50 \%$ response, $M R$ minor response $(<50 \%)$, $N R$ no response, $S D$ stable disease, $R R / U R$ resistant or untreated relapse $(<50 \%$ response)]}

Patients in good response to first-line treatment (CR/VGPR/PR) and sensitive relapse (SR) are good indications for most high-risk solid tumour patients, while patients with stable disease or minor response $(<50 \%)$ (SD/MR) should only be considered for well-defined phase I/phase II trials. Patients with no response (NR) or tumour progression or resistant relapse (RR) have a very short life expectancy even after HDT/HSCT and thus should not be considered.

Age (see Table 92.3). Age plays a crucial role for outcome predictions. Adolescent age is generally associated with inferior outcome. While age $<10$ years is a favourable factor in sarcomas (Ewing tumours and rhabdomyosar-

Table 92.2 EBMT database outcomes after HDT/ASCT in paediatric solid tumours

\begin{tabular}{|l|l|l|l|l|l|l|l|l|l|}
\hline & \multicolumn{3}{|c}{ All patients } & \multicolumn{3}{l|}{ First line patients } & \multicolumn{2}{l|}{ Relapsed patients } & \\
\hline Diagnoses & Patients & 5-years pSU & 5-years pEFS & Patients & 5-years pEFS & Patients & 5-years pEFS & $p$-value \\
\hline Neuroblastoma & 7303 & $0.44 \pm 0.01$ & $0.36 \pm 0.01$ & 5993 & $0.37 \pm 0.01$ & 432 & $0.25 \pm 0.02$ & $<0.001$ \\
\hline Ewing tumors & 2886 & $0.47 \pm 0.01$ & $0.40 \pm 0.01$ & 2101 & $0.44 \pm 0.01$ & 483 & $0.29 \pm 0.02$ & $<0.001$ \\
\hline Wilms tumors & 601 & $0.55 \pm 0.02$ & $0.51 \pm 0.02$ & 208 & $0.52 \pm 0.04$ & 345 & $0.53 \pm 0.03$ & 0.567 \\
\hline Soft tissue sa. & 1097 & $0.29 \pm 0.02$ & $0.24 \pm 0.01$ & 673 & $0.26 \pm 0.02$ & 260 & $0.20 \pm 0.03$ & 0.003 \\
\hline Brain tumors & 2733 & $0.45 \pm 0.01$ & $0.39 \pm 0.01$ & 2020 & $0.43 \pm 0.01$ & 444 & $0.22 \pm 0.02$ & $<0.001$ \\
\hline Germ cell tu. & 529 & $0.59 \pm 0.03$ & $0.49 \pm 0.02$ & 270 & $0.50 \pm 0.03$ & 201 & $0.44 \pm 0.04$ & 0.083 \\
\hline Retinoblastomas & 162 & $0.64 \pm 0.04$ & $0.59 \pm 0.04$ & 98 & $0.64 \pm 0.05$ & 53 & $0.50 \pm 0.07$ & 0.099 \\
\hline Kidney tumors & 73 & $0.49 \pm 0.07$ & $0.48 \pm 0.07$ & 30 & $0.51 \pm 0.10$ & 30 & $0.48 \pm 0.10$ & 0.615 \\
\hline Osteosarcomas & 290 & $0.28 \pm 0.03$ & $0.24 \pm 0.03$ & 129 & $0.34 \pm 0.05$ & 128 & $0.13 \pm 0.03$ & $<0.001$ \\
\hline Miscellaneous & 784 & $0.40 \pm 0.02$ & $0.33 \pm 0.02$ & 511 & $0.34 \pm 0.02$ & 146 & $0.21 \pm 0.04$ & 0.057
\end{tabular}

${ }^{a}$ Log-rank test: first line versus relapse patients 
Table 92.3 EBMT database outcomes after HDT/ASCT in paediatric solid tumours according to patient age

\begin{tabular}{|l|r|r|r|r|r|r|r|l|} 
& \multicolumn{4}{|c|}{ Age $\leq$ years } & \multicolumn{3}{l|}{$5<$ age $\leq 10$ years } & \multicolumn{1}{l|}{$10<$ age $\leq 18$ years } \\
\hline Diagnoses & Patients & Patients & 5 -years pEFS & Patients & 5 -years pEFS & Patients & 5 -years pEFS & $p$-value \\
\hline Neuroblastoma & 7303 & 5267 & $0.41 \pm 0.01$ & 1742 & $0.26 \pm 0.01$ & 361 & $0.18 \pm 0.03$ & $<0.001$ \\
\hline Ewing tumors & 2886 & 388 & $0.38 \pm 0.03$ & 622 & $0.47 \pm 0.02$ & 1913 & $0.39 \pm 0.01$ & 0.001 \\
\hline Wilms tumors & 601 & 201 & $0.57 \pm 0.04$ & 318 & $0.49 \pm 0.03$ & 96 & $0.43 \pm 0.06$ & 0.173 \\
\hline Soft tissue sa. & 1097 & 286 & $0.30 \pm 0.03$ & 287 & $0.29 \pm 0.03$ & 453 & $0.17 \pm 0.02$ & $<0.001$ \\
\hline Brain tumors & 2733 & 1243 & $0.44 \pm 0.02$ & 799 & $0.37 \pm 0.02$ & 751 & $0.33 \pm 0.02$ & 0.039 \\
\hline Germ cell tu. & 529 & 165 & $0.52 \pm 0.04$ & 47 & $0.41 \pm 0.08$ & 328 & $0.49 \pm 0.03$ & 0.273 \\
\hline Retinoblastomas & 162 & 132 & $0.58 \pm 0.05$ & 26 & $0.69 \pm 0.10$ & 10 & $0.50 \pm 0.18$ & 0.727 \\
\hline Kidney tumors & 73 & 33 & $0.51 \pm 0.10$ & 22 & $0.53 \pm 0.11$ & 12 & $0.36 \pm 0.15$ & 0.631 \\
\hline Osteosarcomas & 290 & 4 & $0.75 \pm 0.22$ & 35 & $0.29 \pm 0.08$ & 248 & $0.22 \pm 0.03$ & 0.260 \\
\hline Miscellaneous & 784 & 342 & $0.40 \pm 0.03$ & 145 & $0.23 \pm 0.05$ & 302 & $0.29 \pm 0.03$ & 0.016 \\
\hline
\end{tabular}

coma), neuroblastoma has an earlier cut-off at 5 years. Patients with neuroblastoma $\leq 18$ months at diagnosis need biological profiling and are only eligible with high-risk biological features, in particular MYCN amplification (Canete et al. 2009).

Double HSCT approaches. The EBMT data on repetitive HDT/HSCT approaches shows no advantage over single HDT/HSCT. However, the elective selection of particular poorprognosis patients in phase II settings is a likely bias. Recent data from a randomized trial in neuroblastoma emerged with superiority for the tandem strategy for high-risk neuroblastoma front-line patients (Park et al. 2016).

Busulfan-melphalan. This HDT combination is the only one in the EBMT database resulting in significantly improved survival rates in neuroblastoma and Ewing tumours.

Allo-HSCT. No advantage for allo-HSCT can be detected in the EBMT data for any paediatric solid tumour indication. The potential bias of negative selection of particular poor-prognosis patients needs to be considered.

\subsubsection{Neuroblastoma}

High-risk neuroblastoma (NBL) is defined by widespread disease $>18$ months, including any stage and age in the presence of MYCN oncogene amplification (Cohn et al. 2009; Moroz et al. 2011). In 2018 standard treatments include multicycle induction, extensive surgery to the primary tumour site, HDT/auto-HSCT, at least local radiotherapy and maintenance with 13-cis reti- noid acid, ideally with ch14.18 antibody-based immunotherapy in addition (Park et al. 2016; Yu et al. 2010; Ladenstein et al. 2017, 2018).

Age $<18$ months is an important prognostic discriminator (Cohn et al. 2009; Moroz et al. 2011). The European high-risk NBL study (HR-NBL-1/SIOPEN) randomized BU/MEL and CEM (CBP, VP, MEL) after an adequate response to COJEC induction. BU/MEL HDT resulted in a better EFS and OS with fewer severe adverse events than CEM reporting a 3 -year EFS of $50 \%$ (95\% CI 45-56) for BU/ MEL versus 38\% (32-43) for CEM prior immunotherapy was available for all trial patients in Europe (Ladenstein et al. 2017).

After decades of dose escalation being explored by many teams, the randomized results of the ANBL0532 COG as presented at ASCO 2016 finally found superiority for double HDT/ HSCT [1st HDT, CY and TT; 2nd HDT, CBP, VP and MEL (with reduced doses of single HDT CEM) vs. single HDT (CEM)/auto-HSCT (Park et al. 2016)]. The 3-year EFS and OS were significantly higher for tandem transplant patients $(73.2 \% ; 85.6 \%)$ in comparison with the single transplant arm (55.5\%; 75.8\%). Anti-GD2 antibody-based immunotherapy was beneficial for both arms (Yu et al. 2010).

Targeted therapies, in particular iodine-131metaiodobenzylguanidine (mIBG) therapy with and without chemotherapy and/or HDT followed by HSCT, have generated increasing interest (Lee et al. 2017; Johnson et al. 2011).

Further HDT tandem approaches with or without $\mathrm{mIBG}$ are currently explored by the COG as well as SIOPEN in planned randomized 
trials. Published mIBG treatments refer to tandem $\mathrm{mIBG}$ in rapid sequence, to $\mathrm{mIBG}$ as component of HDT (CEM or BU/MEL) and $\mathrm{mIBG}$ combination therapies. They vary in dose, single vs. tandem schedules applied and various combinations with chemotherapy (topotecan or irinotecan and VCR) or radiation sensitizers (i.e. vorinostat increasing $\mathrm{mIBG}$ uptake).

In relapse patients the EBMT data (Ladenstein et al. 1993) showed a benefit from salvage HDT/ HSCT in responding patients relapsing after 12 months from diagnosis and without a previous HDT.

Allo-HSCT as immunotherapy received special attention after introduction of RIC and NMA transplants. Some reports highlight a graftversus-tumour (GvT) effect with adopted alloHSCT approaches, while the EBMT data shows no benefit with classical allo-HSCT (Ladenstein et al. 1994). Research on experimental approaches to allogeneic HSCT is ongoing. Haplo-HSCT is a feasible option in very high-risk $\mathrm{R} / \mathrm{R}$ patients capable to induce long-term remission in some with tolerable side effects allowing addition of post-transplant immune adoptive strategies (Illhardt et al. 2018).

\subsubsection{EBMT Data (See Tables 92.2 and} 92.3)

Median age of 7672 patients is 3.7 years $(7,504$ auto-HSCT, 168 allo-HSCT). EBMT registry data pointed at an early stage to the superiority of the BU/MEL HDT (Ladenstein et al. 2017). A multivariate analysis of the EBMT data found significantly better EFS associated with age $<2$ years at ASCT $(p<0.0001)$, a good remission status $(\geq \mathrm{PR})$ before HDT/HSCT $(p<0.0001)$, use of PBSC $(p=0.014)$, ASCT (vs. allo-HSCT) $(p=0.031)$, as well as with the BU/MEL combination for HDT $(p<0.01)$ (Ladenstein et al. 2008).

\subsubsection{Indications $\mathbf{2 0 1 8}$}

Standard indications include first-line high-risk NBL >18 months at diagnosis with widespread metastatic disease or those of any age with MYCN amplified tumours with INSS stages 2-4. Any responding metastatic relapse in patients $>18$ months and any MYCN amplified tumour without prior HDT/HSCT are good indications. Any other indication is reserved for well-designed experimental phase I/phase II trials. Children $<18$ months need to be evaluated for a high-risk biological risk profile prior to being considered for HDT/auto-HSCT (Canete et al. 2009; Moroz et al. 2011; Cohn et al. 2009).

\subsubsection{Ewing Tumours}

A number of publications proposed a potential role of HDT/HSCT in Ewing tumours (Ladenstein et al. 2010; Luksch et al. 2012) during primary treatments for patients with multifocal bone or BM metastases, whereas indications for those with lung metastases is more questionable (Burdach and Jürgens 2002). Some publications found BU/MEL HDT more active in comparison to other HDT regimes (Tenneti et al. 2018). TBI was investigated (Burdach and Jürgens 2002) without any clear benefit but high toxicity and mortality.

The Euro-EWING 99 study group (Ladenstein et al. 2010) published the largest population of HSCT on primary disseminated multifocal Ewing sarcomas (PDMES) with 281 patients receiving BU/MEL HDT/auto-HSCT after VIDE (VCR, IFO, DOX, VP) induction. The 3-year EFS and OS were $27 \pm 3 \%$ and $34 \pm 4 \%$ and were $45 \%$ for patients $\leq 14$ years. Patients $\geq 14$ years $(\mathrm{HR}=1.6)$, with a primary tumour volume $>200 \mathrm{~mL}(\mathrm{HR}=1.8)$, more than one bone metastatic site $(\mathrm{HR}=2.0), \mathrm{BM}$ metastases $(\mathrm{HR}=1.6)$ and additional lung metastases ( $\mathrm{HR}=1.5)$, carry an increased risk at diagnosis. A score based on these factors identified patients with an EFS rate of $50 \%$ for scores $\leq 3$ (82 patients), $25 \%$ for a score $>3$ and $\leq 5$ (102 patients) and $10 \%$ for score $\geq 5$ (70 patients; $p<0.0001$ ).

In 2018 the Euro-EWING 99 study group published the results of the randomized comparison of BU/MEL over standard chemotherapy with VAI (7 courses) which was offered to patients if aged $<50$ with poor histologic response ( $\geq 10 \%$ viable cells) after VIDE induction (6 courses) or large tumour volume at diagnosis $(\geq 200 \mathrm{~mL})$. The risk of event was significantly decreased by BU/MEL compared 
to VAI (VCR, actinomycin, IFO) ( $\mathrm{HR}=0.64$, 95\% CI $=0.43-0.95 ; p=0.026) ; 3$ - and 8-year EFS were, respectively, $69.0 \%(60.2-76.6 \%)$ versus $56.1 \%(47.6-65.4 \%)$ and $60.7 \%(51.1-$ $69.6 \%$ ) versus $47.1 \%(37.7-56.8 \%)$. OS results also favoured BU/MEL. For this group of patients, BU/MEL is now a standard of care (Whelan 2018).

Tandem HDT and allo-HSCT were part of the EICESS92 and Meta-EICESS protocols yielding long-term DFS in patients with advanced Ewing tumours (Burdach and Jürgens 2002).

Patients suffering a relapse generally have a poor prognosis with conventional chemotherapy. The role of HDT/auto-HSCT still awaits clarification in randomized controlled studies (Tenneti et al. 2018; Ferrari et al. 2015). A GvT or improved survival following allo-HSCT effect was highlighted in some reports, but a retrospective review (Thiel et al. 2011) could not identify benefits with either RIC or MAC or with either HLA-matched or HLA-mismatched grafts.

\subsubsection{EBMT Data (See Tables 92.2} and 92.3)

The median age of 3019 (2970 autologous, 49 allogeneic) evaluable Ewing tumour patients is 12.5 years with 2161 patients who received HDT during primary treatment and 498 after relapse (disease status not specified in 360). BU/MEL HDT achieved better results in firstline settings, while TBI clearly was associated with poorer outcomes. Multivariate analysis of the EBMT data showed increased risks for patients $>14$ years, a remission status less than CR1, PR or CR2, BM as stem cell source and HDT other than BU/MEL.

\subsubsection{Indications $\mathbf{2 0 1 8}$}

BU/MEL HDT for patients with a poor histological response after induction and/or a tumour volume $\geq 200 \mathrm{~mL}$ is now standard of care. Patients with primary metastatic disease at sites other than the lungs and a low-risk score may be considered good candidates in the absence of a controlled trial. Any metastatic relapse without prior HDT may be considered for controlled phase II HDT protocols.

\subsubsection{Soft Tissue Sarcoma (STS)}

Rhabdomyosarcoma (RMS) is the most common STS of childhood. A multivariate analysis of 269 patients with metastatic RMS found age $(>10$ years, $p<0.0001)$ and bone/BM involvement $(p<0.019)$ to be the most important predictors for fatal outcome (Thiel et al. 2013). In STS dose escalation with auto-HSCT produced only short-lived remissions with generally disappointing outcome data unless patients were chemosensitive and receiving HDT as consolidation in CR (Admiraal et al. 2010). Results of a recent review do not justify the use of HDC/HSCT as standard therapy for children with metastatic RMS (Admiraal et al. 2010).

A systematic review of HDT/auto-HSCT in locally advanced or metastatic nonrhabdomyosarcoma soft tissue sarcoma (NRSTS) was undertaken and evaluated 294 patients with 19 different subtypes of malignant NRSTS in 62 studies covering an age span between 10 and 46 years. This analysis found no advantage for HDT over standard chemotherapy (Peinemann and Labeit 2014).

The use of allo-HSCT in patients with advanced RMS is still experimental. No patients with residual disease before allo-SCT were converted to CR. In a subset of patients, it may constitute a valuable approach for consolidating $\mathrm{CR}$, but this awaits prospective validation (Thiel et al. 2013).

\subsubsection{EBMT Data (See Tables 92.2} and 92.3)

The median age of 1116 evaluable patients (1035 autologous, 81 allogeneic) is 9 years with 718 receiving HDT during primary treatment and 275 after relapse (disease status not specified in 123 patients).

\subsubsection{Indications $\mathbf{2 0 1 8}$}

Currently there is no evidence-based standard indication for HDT/HSCT in STS.

\subsubsection{Brain Tumours}

Paediatric embryonal brain tumour patients treated with craniospinal irradiation (CSI) are at 
risk for adverse effects, with greater severity in younger patients. Two publications in 2017 refer to this situation. Outcomes of CSI vs. HDT/auto-HSCT and delayed CSI were compared in 201 newly diagnosed patients [medulloblastoma $(72 \%)$, supratentorial primitive neuroectodermal tumour (sPNET; 18\%) or pineoblastoma (10\%)]. Outcomes with adjuvant HD/HSCT followed by delayed CSI are comparable to upfront CSI for young paediatric embryonal brain tumour patients, but benefits regarding neurocognitive outcomes await future assessment (Raleigh et al. 2017).

The second report summarizing HDT/HSCT (29 with 3 tandem transplants and 15 with 2 tandem transplants) as irradiation-avoiding or irradiation-minimizing approach reported encouraging irradiation-free survival in children with newly diagnosed malignant brain tumours [medulloblastoma/primitive neuroectodermal tumour (21 patients), atypical teratoid/rhabdoid tumour (8), high-grade glioma (5), malignant germ cell tumour (4), ependymoma (3) and choroid plexus carcinoma (3)] (Guerra et al. 2017). The PFS and OS for newly diagnosed patients were $68.9 \pm 9.9 \%$ and $73.5 \pm 9.3 \%$, while it was only $11.8 \pm 9.8 \%(p<0.001)$ and $15.1 \pm 12.3 \%$ $(p=0.0231)$ at relapse, respectively.

The Children's Cancer Group 99703 study assessed 92 patients receiving 3 consolidation cycles of HDT (TT and CBP) and auto-HSCT after biopsy/resection and 3 conventional-dose chemotherapy cycles. Response rates to induction and consolidation were high in patients with residual tumour with $73.3 \%$ and $66.7 \%$, respectively, resulting in 5-year EFS and OS of $43.9 \pm 5.2 \%$ and $63.6 \pm 5 \%$. Gross total resection versus less than gross total resection was the only significant outcome comparison (Cohen et al. 2015).

The HIT97 national trial tested a stratified relapse protocol using either intensive chemotherapy, potentially high dose, or oral chemotherapy. Adding HDT in patients who responded to the initial courses of chemotherapy did not improve survival. Patients with relapsed cerebral PNET responding to conventional chemotherapy did not profit from HDT (Bode et al. 2014).

\subsubsection{EBMT Data (See Tables 92.2} and 92.3)

The median age for 2818 evaluable brain tumour patients (2809 autologous, 9 allogeneic) is 6 years with 2039 receiving HDT during primary treatments and 442 after relapse (disease status not specified in 337).

Medulloblastoma is the leading indication with $69 \%$ followed by astrocytoma (7\%), glioblastoma (7\%), ependymoma (6\%) and other nonspecified CNS tumours (11\%). Medulloblastoma patients' outcome is better when submitted to HDT/SCT in first remission and if younger $<3$ years. A more detailed analysis by various brain tumours histologies and distinct risk factors is not possible in the current dataset.

\subsubsection{Indications $\mathbf{2 0 1 8}$}

Patients with high-risk medulloblastoma (primary metastases/relapse) of any age older than 3 years are eligible for HDT/HSCT in combination with radiation, while in infants HDT/HSCT is used with the aim of reducing (volumes and doses) or avoiding radiation (Finlay and Massimino 2010).

Metastatic PNETs at diagnosis or with additional high-risk features such as incomplete resection or young age (younger than 3 or 5 years) as well as infants and young children ( $<4$ years) with malignant brain tumours are further indications. Very controversial indications include high-grade glioma. Currently there is little or no indication for HDT/HSCT in ependymoma, brain stem glioma or pineoblastoma. More investigations are required to define the optimal HDT for each tumour type. Most groups use similar HDT regimens, i.e. BU/TT (SFOP, Spain), VP/TT/CBDCA (US/CCG, Germany, Spain) or a tandem approach Vp16/CBDCA - TTP/L-PAM (Italy).

\subsubsection{Wilms' Tumour (WT)}

Pooling data of 19 publications (5 HDT, 6 NoHDT, 8 both), an advantage to HDT with a hazard ratio (HR) of 0.87 for EFS and 0.94 for OS was observed. Further analyses of risk groups, defined by treatment and/or histology prior to first relapse, 
suggested a HR for EFS of 0.90 for those of highand 0.50 for the very high-risk patients. However, a great uncertainty persists in the absence of randomized trials concerning the role of HDT following relapse after treatment for WT (Ha et al. 2013).

The CIBMTR retrospective analysis on 253 relapsed WT receiving HDT/auto-HSCT reports 5 -year EFS and OS rates of 36\% (95\% CI, $29-43 \%$ ) and $45 \%$ (95\% CI, 38-51\%), respectively. Outcomes are similar to those reported in the literature. So far attempts to conduct a randomized trial comparing maintenance chemotherapy with consolidation versus HDT/ auto-HSCT have failed in this indication (Malogolowkin et al. 2017).

\subsubsection{EBMT Data (See Tables 92.2} and 92.3)

The median age in 625 patients with auto-HSCT (212 HDT during primary treatment; 348 after relapse) is 6 years. Reported superior results for patients receiving MEL alone may be correlated to the favourable response status prior HDT: $76 \%$ in CR1 and $64 \%$ in CR2.

\subsubsection{Indications $\mathbf{2 0 1 8}$}

Experience of the SIOP, GPOH, NWTS, MRC and respective national groups over the last 20 years found the probability of cure of $30 \%$ at best in the presence of adverse prognostic factors. High-risk factors are unfavourable histology and metastatic disease (Presson et al. 2010) and are after relapse again unfavourable histology and one of the following criteria: extra-pulmonary relapse or abdominal relapse after radiation, stage IV, more than two drugs in the first-line regimen or relapse within 1 year. HDT is indicated if a response to second-line treatment is achieved.

\subsubsection{Germ Cell Tumours}

CNS germ cell tumours (GCTs) can be divided into major groups including germinomas (having a superior prognosis) and non-germinomatous GCTs (NGGCTs), with teratomas often considered a separate category- and represent approximately $3 \%$ of primary paediatric brain tumours.
NGGCTs are less radiosensitive, but adjuvant chemotherapy improved survival.

CNS GCT relapses commonly occur at the primary tumour site, and in about $30 \%$ there is concomitant leptomeningeal spread. Salvage therapies include additional surgery, focal or CSI and HDT/ auto-HSCT. For pure germinoma patients previously receiving radiation therapy, HDT/autoHSCT is often recommended. HDT/HSCT showed curative potential for some relapsed systemic NGGCTs, in particular for those achieving a CR to chemotherapy (Echevarria et al. 2008).

The management of intracranial GCTs is complex because of varied clinical presentations, tumour sites, treatments and outcomes and the need for multidisciplinary input.

In an international Delphi approach, key areas of consensus were defined to guide and streamline clinical management of patients with intracranial germ cell tumours (Murray et al. 2015).

\subsubsection{EBMT Data (See Tables 92.2} and 92.3)

The median age of 553 patients with HDT/ASCT (270 during primary treatment, 211 after relapse, disease status not reported in 72) registered as GCT and considered high risk is 13 years. However, differentiation of subgroups is poor.

\subsubsection{Indications $\mathbf{2 0 1 8}$}

As paediatric patients with extracranial GCTs may expect an excellent outcome with conventional chemotherapy approaches, there is no standard indication for HDT/ASCT.

High-risk patients with extracranial GCTs are initial nonresponders or poor responders (no local control achieved) and patients after relapse failing to achieve second CR. In high-risk CNS GCT patients $<18$ years, the following criteria for HDT may be adopted: recurrent CNS GCT when biological remission is achieved prior to HDC and insufficient response to primary chemotherapy.

\subsubsection{Osteosarcoma}

Even in responding high-risk patients treated with HDT/HSCT in first or second remission, the 
length of remission is short, and relapse occurs early after HDT. High-risk features include poor histological response or nonresponse of the primary tumour at the time of definitive surgery, inoperable, axial tumours (large volume), primary dissemination or relapse other than isolated, late lung metastases.

The French Society of Paediatric Oncology (SFCE) explored HDT with high-dose TT and auto-HSCT in 45 children with relapsed osteosarcoma (median age 15.9 years) after initial progression of metastatic disease (2), first relapse (26) and second or third relapse (17). Three-year OS was $40 \%$ and 3-year PFS 24\%. A randomized study for recurrent osteosarcoma between standard salvage chemotherapy and high-dose TT with auto-HSCT is ongoing (Marec-Berard et al. 2014).

Earlier reports did not encourage HDT regimens (Sauerbrey et al. 2001) and included MEL and VP, with additional CBP by the Cooperative Osteosarcoma Study Group, or two courses of high-dose VP and CBP by the Italian Sarcoma Group (Boye et al. 2014; Fagioli et al. 2002).

\subsubsection{EBMT Data (See Tables 92.2} and 92.3)

The median age in 294 evaluable patients (134 HDT during primary treatment; 130 after relapse, disease status not specified in 30; 288 autologous, only 6 allogeneic) is 14.3 years.

\subsubsection{Indications $\mathbf{2 0 1 8}$}

There is no standard indication for HSCT based on published results or EBMT data.

\subsubsection{Retinoblastoma}

There is one systematic review on HDT/autoHSCT based on 15 studies and a total of 101 patients (Jaradat et al. 2012). Following treatment for metastatic and relapsed disease, 44 of 77 patients $(57.1 \%)$ were alive with no evidence of disease at the time of follow-up. However, a higher rate of local relapse developed in patients with CNS metastases (73.1\%), which dropped to $47.1 \%$ in patients who received thiotepa. In patients with trilateral or bilateral advanced retinoblastoma, five of seven $(71.4 \%)$ with reported outcome data as well as patients with tumour at the surgical margin of the optic nerve and/or extrascleral extension, six of seven patients $(85.7 \%)$ were alive without evidence of disease at last follow-up. Durable tumour control was achieved in patients with non-CNS metastases, trilateral or bilateral advanced retinoblastoma, and with tumour at the surgical margin of the optic nerve and/or extrascleral extension. Patients with CNS metastases require TT to improve tumour control (Dunkel et al. 2010).

Advocated HDT/auto-HSCT approaches are CARBOPEC (CBP, VP, CY) (Jaradat et al. 2012), but for CNS-positive patients, TT or BU was introduced. Other groups used combinations including MEL and CBDCA and/or VP for metastatic retinoblastoma and reported promising survival results for patients without CNS involvement.

\subsubsection{EBMT Data (See Tables 92.2}

and 92.3)

The median age for 170 evaluable patients (99 during primary treatment ( $40 \%$ with localized/ regional disease; $60 \%$ with metastatic disease); 54 after relapse; 17 disease status not specified; 169 autologous, 1 allogeneic) is 3.5 years.

\subsubsection{Indications 2018}

Future trials should take the following high-risk factors into consideration: involvement of the cut end or subarachnoidal space of the optic nerve after enucleation, orbital involvement and distant metastatic disease and CNS disease.

\section{Key Points}

- In neuroblastoma and Ewing sarcoma, there is clear evidence for the advantage of HDT/auto-HSCT with an increasing interest in tandem transplants.

- In other paediatric solid tumour, indication still lacks randomized trials, and indications are based on observational studies, case reports and EBMT database only. 


\subsection{Solid Tumours in Adults}

Francesco Lanza

\subsubsection{Auto-HSCT}

Supported by a strong rationale from laboratory studies and apparently "convincing" results of early phase II studies, in the 1990s auto-HSCT was uncritically adopted as a potentially curative option for solid tumours. For this reason, randomized trials comparing high-dose therapy with conventional control arm were difficult to conduct. As a result, the number and size of clinical studies initiated (and often abandoned before completion) to prove or disprove its value were largely insufficient. Nowadays, after 25 years of clinical research and thousands of patients receiving auto-HSCT, the benefit of autotransplant in solid tumours, with the possible exception of selected patients with breast cancer (BC) and germ cell tumours (GCTs), is still unsettled (Fig. 92.1) (Sureda et al. 2015).

\subsubsection{Breast Cancer (BC)}

The role of auto-HSCT for primary breast cancer at high risk of recurrence (at least four involved axillary lymph nodes) has been assessed by several randomized trials, recently evaluated by a meta-analysis of individual patient data (Berry et al. 2011; Pedrazzoli et al. 2015). Overall, it was shown that auto-HSCT prolonged DFS when used as adjuvant therapy and showed a benefit on BC-specific survival and OS in selected cohorts of patients (Nitz et al. 2005; Pedrazzoli et al. 2015).

Whether auto-HSCT has benefit in the context of contemporary taxane-based regimens and targeted therapies is largely unknown. Seven phase III studies have been published in peer-reviewed journals. Most of these trials showed improved PFS in the auto-HSCT arm but only one OS advantage. Six randomized trials, including 866 metastatic breast cancer (MBC) patients, have been analysed in the parallel meta-analysis of individual patient data (Berry et al. 2011) showing a significant improvement in PFS but no improvement in OS.
Overall, based on the randomized studies so far, meta-analyses and retrospective studies, autoHSCT may still represent a therapeutic option for younger patients harbouring HER2-negative tumours and having gross involvement of axillary nodes (adjuvant setting) or highly chemosensitive disease (advanced setting) (Martino et al. 2016).

\subsubsection{Germ Cell Tumours (GCTs)}

Auto-HSCT is not recommended as first-line therapy in GCT. In relapsed GCT high-dose therapy is considered a therapeutic option, especially when poor prognostic factors are present (Lorch et al. 2011; Necchi et al. 2015; De Giorgi et al. 2017a; b). A randomized study (Tiger study) comparing conventional-dose therapy with highdose therapy is ongoing. Auto-HSCT is a standard of care for patients that are (primary) refractory to platinum-based chemotherapy or for those with a second or further relapse (Necchi et al. 2015). Multiple intensified cycles with $\mathrm{CBP} / \mathrm{VP}$ are recommended as the standard HDT for GCT also due to concerns that using a threedrug regimen would require dose reductions of the two most active drugs in this disease.

Furthermore, auto-HSCT can be safely administered in high-risk patients older than 45 years. However, since the prognosis is poorer for older patients with non-seminoma histology, a comprehensive risk-benefit evaluation should include co-morbidities and the patient's risk category.

The assessment of a large series of EBMT centres, including 46 cases with pure seminoma, seems to support the notion that auto-HSCT may represent a valuable therapeutic option after standard-dose chemotherapy failure in this patient category (Necchi et al. 2017).

EBMT conducted a retrospective analysis on 29 patients with refractory gestational trophoblastic neoplasia (GTN) treated with autoHSCT. Data showed that HDT based on CBP seems to be active in this heavily pretreated patient population and that auto-HSCT represents a possible option for patients with refractory GNT (Necchi et al. 2016).

The role of auto-HSCT in mediastinal nonseminoma (MnS) GCT disease category is under evaluation. Data from EBMT confirmed that the 
$\mathrm{MnS}$ was characterized by the poorest outcome with 5-year OS ranging from $40 \%$ to $45 \%$. The use of auto-HSCT as both early intensification and at disease recurrence proved to be effective, given upfront, and may produce a 15-20\% absolute improvement in survival compared with standard-dose CT.

\subsubsection{Soft Tissue Sarcoma (STS)}

STS accounts for about $1 \%$ of adult cancers. Based on the observation of a dose-response correlation for some drugs used in STS, e.g. DOX and IFO, HDT with auto-HSCT has been investigated in some, mostly non-randomized phase II trials. Most of these trials found few patients to possibly benefit from auto-HSCT but, owing to the small patient numbers of each of the included histologic subgroups, could not establish robust markers for identifying these patients. A recent meta-analysis on this subject found no evidence for a benefit of auto-HSCT but, again, did not sufficiently report on outcomes in the different histologic subgroups. However, taking into account that the current WHO classification differentiates more than 50 histological subtypes of STS, it might be hypothesized that clinical response to auto-HSCT may vary significantly from different histological varieties.

\subsubsection{Other Solid Tumours}

Data from randomized phase III studies comparing HDT vs. conventional-dose chemotherapy for first-line treatment of advanced ovarian cancer and limited or extensive small cell lung cancer have shown no statistically significant difference in PFS or OS. Limitations due to study design, difficulty in recruitment and toxicity may have accounted for the lack of favourable results that were expected based on previous phase II and retrospective analyses of such highly chemosensitive diseases.

In other chemosensitive histologies, including sarcomas and CNS tumours, data regarding autoHSCT in adult patients are limited, again based on clinical trials without randomization and retrospective analyses. For this reason, auto-HSCT cannot be recommended as standard of care. High-dose therapy can be regarded as a potential clinical option in selected patients with Ewing's sarcoma and medulloblastoma.

\subsubsection{Allo-HSCT}

Immune therapy for cancer is being pursued with extraordinary interest by researchers all over the world, given the recent scientific acquisitions on immune mechanisms that control cancer and the introduction in the marketplace of checkpoint inhibitor molecules, such as nivolumab/pembrolizumab (PD-1/PDL-1 inhibitors), ipilimumab (anti-CTLA4), etc. The paradigm for immune therapy of cancer is allo-HSCT, whose therapeutic effect is carried out by immunocompetent $\mathrm{T}$ cells of the donor, an effect known as GvT or graft-versus-tumour effect. Several studies of allo-HSCT in selected solid tumours, namely, renal cell cancer (RCC), ovarian cancer, BC, colorectal cancer and others, with some evidence of GvT and occurrence of transplant-related toxicities, mostly GvHD have been reported. In RCC, a long-term survival effect in a fraction (20\%) of patients was documented. Since 2004, when molecularly targeted drugs were introduced into the clinic for renal cell cancer, patient referral for transplant dropped precipitously, and transplant rate evaluation for solid tumours from 2009 was limited to a few patients in Europe.

A recent survey provided a picture of the status of allo-HSCT for solid tumours in EBMT centres (Bregni et al. 2016). In contrast to our expectations, allo-HSCT for solid tumour indications has not been abandoned, even if its use has been markedly reduced. Based on these findings, allograft for solid tumours as adoptive immunotherapy is still being used in EBMT centres, in a few institutions and in a few selected indications, thus confirming the willingness of several centres to share data, adopt common protocols and continue to use this strategy in highly selected cases.

\subsubsection{Renal Cell Cancer}

There is no clinical experience of allografting in $\mathrm{RCC}$, that is, TKI and mTOR refractory; nowadays, allo-HSCT should be considered only in the context of prospective studies. 


\subsubsection{Soft Tissues Sarcomas}

The use of allo-HSCT has been investigated in paediatric sarcoma and rhabdomyosarcoma. Original data on allo-HSCT in adult STS is rare and stems from small case series (Sureda et al. 2015). In 2007, a retrospective study of the EBMT investigated the use of reducedconditioning allo-HSCT in 14 STS patients. The authors concluded that an immune-mediated effect might have contributed to tumour control in some patients. Interestingly, the low number of patients examined did not permit a histotypestratified analysis.

\section{Key Points}

- The benefit of auto-HSCT in solid tumours of the adults, with the possible exception of selected patients with breast cancer and germ cell tumours, is still unsettled.

- Despite the great potential, cell therapy programmes for cancer control still have a marginal role in the management of patients with solid tumours. This issue should be regarded as a priority for medical oncology and cell therapy/ transplantation societies, also in view of the recent development of immune checkpoint inhibitors that represent a major breakthrough in cancer treatment and may well be incorporated in cell therapy programmes.

- The story of HSCT in solid tumours demonstrates the importance of adopting an internationally co-ordinated approach to the investigation of this treatment modality. There needs to be an increased emphasis on prospective trials that are statistically robust and have well-defined criteria for patient selection. Only these will be able to demonstrate whether HSCT, alone or incorporated into programmes with novel therapeutic modalities, is worthwhile in patients for whom conventional treatments have often limited impact on survival.

\section{References}

Admiraal R, van der Paardt M, Kobes J, et al. High-dose chemotherapy for children and young adults with stage IV rhabdomyosarcoma. Cochrane Database Syst Rev. 2010;12:CD006669.

Berry DA, Ueno NT, Johnson MM, et al. High dose chemotherapy with autologous stem cell support versus standard-dose chemotherapy: overview of individual patient data from 15 randomized adjuvant therapy breast cancer trials. J Clin Oncol. 2011;29:3214-23.

Bode U, Zimmermann M, Moser O, et al. Treatment of recurrent primitive neuroectodermal tumors (PNET) in children and adolescents with high-dose chemotherapy (HDC) and stem cell support: results of the HITREZ 97 multicentre trial. J Neuro-Oncol. 2014;120:635-42.

Boye K, Del Prever AB, Eriksson M, et al. High-dose chemotherapy with stem cell rescue in the primary treatment of metastatic and pelvic osteosarcoma: final results of the ISG/SSG II study. Pediatr Blood Cancer. 2014;61:840-5.

Bregni M, Badoglio M, Pedrazzoli P, Lanza F. Is allogeneic transplant for solid tumors still alive? Bone Marrow Transplant. 2016;51:751-2.

Burdach S, Jürgens H. High-dose chemoradiotherapy (HDC) in the Ewing family of tumors (EFT). Crit Rev Oncol Hematol. 2002;41:169-89.

Canete A, Gerrard M, Rubie H, et al. Poor survival for infants with MYCN-amplified metastatic neuroblastoma despite intensified treatment: the International Society of Paediatric Oncology European Neuroblastoma Experience. J Clin Oncol. 2009;27(7):1014-9.

Cohen BH, Geyer JR, Miller DC, et al. Pilot study of intensive chemotherapy with peripheral hematopoietic cell support for children less than 3 years of age with malignant brain tumors, the CCG-99703 phase I/II study. A report from the Children's Oncology Group. Pediatr Neurol. 2015;53:31-46.

Cohn SL, Pearson ADJ, London WB, et al. The International Neuroblastoma Risk Group (INRG) classification system: an INRG task force report. J Clin Oncol. 2009;27:289-97.

De Giorgi U, Nicolas-Virelizier E, Badoglio M, et al. High-dose chemotherapy for adult-type ovarian granulosa cell tumors: a retrospective study of the European Society for Blood and Marrow Transplantation. Int J Gynecol Cancer. 2017a;27:248-51.

De Giorgi U, Richard S, Badoglio M, et al. Salvage high-dose chemotherapy in female patients with relapsed/refractory germ-cell tumors: a retrospective analysis of the European Group for Blood and Marrow Transplantation (EBMT). Ann Oncol. 2017b;28:1910-6.

Dunkel IJ, Chan HSL, Jubran R, et al. High-dose chemotherapy with autologous hematopoietic stem cell rescue for stage $4 \mathrm{~B}$ retinoblastoma. Pediatr Blood Cancer. 2010;55:149-52. 
Echevarria ME, Fangusaro J, Goldman S. Pediatric central nervous system germ cell tumors: a review. Oncologist. 2008;13:690-9.

Fagioli F, Aglietta M, Tienghi A, et al. High-dose chemotherapy in the treatment of relapsed osteosarcoma: an Italian Sarcoma Group Study. J Clin Oncol. 2002;50:2150-6.

Ferrari S, Luksch R, Hall KS, et al. Post-relapse survival in patients with Ewing sarcoma. Pediatr Blood Cancer. 2015;62:994-9.

Finlay JL, Massimino M. A consensus and state-of-theart workshop: marrow ablative chemotherapy with hematopoietic cell rescue for malignant brain tumors of childhood and adolescence. Pediatr Blood Cancer. 2010;54:634.

Guerra JA, Dhall G, Marachelian A, et al. Marrow-ablative chemotherapy followed by tandem autologous hematopoietic cell transplantation in pediatric patients with malignant brain tumors. Bone Marrow Transplant. 2017;52:1543-8

Ha TC, Spreafico F, Graf N, et al. An international strategy to determine the role of high dose therapy in recurrent Wilms' tumour. Eur J Cancer. 2013;49:194-210.

Illhardt T, Toporski J, Feuchtinger T, et al. Haploidentical stem cell transplantation for refractory/relapsed neuroblastoma. Biol Blood Marrow Transplant. 2018;24:1005-12.

Jaradat I, Mubiden R, Salem A, et al. High-dose chemotherapy followed by stem cell transplantation in the management of retinoblastoma: a systematic review. Hematol Oncol Stem Cell Ther. 2012;5: 107-17.

Johnson K, McGlynn B, Saggio J, et al. Safety and efficacy of tandem 131I-metaiodobenzylguanidine infusions in relapsed/refractory neuroblastoma. Pediatr Blood Cancer. 2011;57:1124-9.

Ladenstein R, Lasset C, Hartmann O, et al. Impact of megatherapy on survival after relapse from stage 4 neuroblastoma in patients over 1 year of age at diagnosis: a report from the European Group for Bone Marrow Transplantation. J Clin Oncol. 1993;11:2330-41.

Ladenstein R, Lasset C, Hartmann O, et al. Comparison of auto versus allografting as consolidation of primary treatments in advanced neuroblastoma over one year of age at diagnosis: report from the European Group for Bone Marrow Transplantation. Bone Marrow Transplant. 1994;14:37-46.

Ladenstein R, Pötschger U, Hartman O, Pearson AD, Klingebiel T, Castel V, Yaniv I, Demirer T, Dini G, EBMT Paediatric Working Party. 28 years of highdose therapy and SCT for neuroblastoma in Europe: lessons from more than 4000 procedures. Bone Marrow Transplant. 2008;41(Suppl 2):S118-27. https://doi.org/10.1038/bmt.2008.69.

Ladenstein R, Pötschger U, Le Deley MC, et al. Primary disseminated multifocal Ewing sarcoma: results of the Euro-EWING 99 trial. J Clin Oncol. 2010;28:3284-91.

Ladenstein R, Pötschger U, Pearson ADJ, et al. Busulfan and melphalan versus carboplatin, etoposide, and melphalan as high-dose chemotherapy for high-risk neu- roblastoma (HR-NBL1/SIOPEN): an international, randomised, multi-arm, open-label, phase 3 trial. Lancet Oncol. 2017;18:500-14.

Ladenstein R, Pötschger U, Valteau-Couanet D, et al. Interleukin 2 with anti-GD2 antibody ch14.18/CHO (dinutuximab beta) in patients with high-risk neuroblastoma (HR-NBL1/SIOPEN): a multicentre, randomised, phase 3 trial. Lancet Oncol. 2018. https:// doi.org/10.1016/1470-2045(18)30578-3

Lee JW, Lee S, Cho HW, et al. Incorporation of highdose 131I-metaiodobenzylguanidine treatment into tandem high-dose chemotherapy and autologous stem cell transplantation for high-risk neuroblastoma: results of the SMC NB-2009 study. J Hematol Oncol. 2017; 10:108.

Lorch A, Bascoul-Mollevi C, Kramar A, et al. Conventional-dose versus high-dose chemotherapy as first salvage treatment in male patients with metastatic germ cell tumors: evidence from a large international database. J Clin Oncol. 2011;29:2178-84.

Luksch R, Tienghi A, Hall KS, et al. Primary metastatic Ewing's family tumors: results of the Italian Sarcoma Group and Scandinavian Sarcoma Group ISG/SSG IV Study including myeloablative chemotherapy and total-lung irradiation. Ann Oncol. 2012;23:2970-6.

Malogolowkin MH, Hemmer MT, Le-Rademacher J, et al. Outcomes following autologous hematopoietic stem cell transplant for patients with relapsed Wilms' tumor: a CIBMTR retrospective analysis. Bone Marrow Transplant. 2017;52:1549-55.

Marec-Berard P, Segura-Ferlay C, Tabone MD, et al. High dose thiotepa in patients with relapsed or refractory osteosarcomas: experience of the SFCE Group. Sarcoma. 2014;2014:475067.

Martino M, Lanza F, Pavesi L, et al. High-dose chemotherapy and autologous hematopoietic stem cell transplantation as adjuvant treatment in high-risk breast cancer: data from the European Group for Blood and Marrow Transplantation Registry. Biol Blood Marrow Transplant. 2016;22:475-81.

Matthay KK, Reynolds CP, Seeger RC, et al. Long-term results for children with high-risk neuroblastoma treated on a randomized trial of myeloablative therapy followed by 13-cis-retinoic acid: a children's oncology group study. J Clin Oncol. 2009;27:1007-13.

Moroz V, Machin D, Faldum A, et al. Changes over three decades in outcome and the prognostic influence of age-at-diagnosis in young patients with neuroblastoma: a report from the International Neuroblastoma Risk Group Project. Eur J Cancer. 2011;47:561-71.

Murray MJ, Bartels U, Nishikawa R, et al. Consensus on the management of intracranial germ-cell tumours. Lancet Oncol. 2015;19:e470-7.

Necchi A, Lanza F, Rosti G, European Society for Blood and Marrow Transplantation, Solid Tumors Working Party (EBMT-STWP) and the Italian Germ Cell Cancer Group (IGG), et al. High-dose chemotherapy for germ cell tumors: do we have a model? Expert Opin Biol Ther. 2015;15:33-44. 
Necchi A, Miceli R, Bregni M, et al. Prognostic impact of progression to induction chemotherapy and prior paclitaxel therapy in patients with germ cell tumors receiving salvage high-dose chemotherapy in the last 10 years: a study of the European Society for Blood and Marrow Transplantation Solid Tumors Working Party. Bone Marrow Transplant. 2016;51: 384-90.

Necchi A, Lo Vullo, Bregni M-et al. Salvage high-dose chemotherapy for relapsed pure seminoma in the last 10 years: results from the European Society for Blood and Marrow Transplantation series 2002-2012. Clin Genitourin Cancer 2017; 15: 163-167.

Nitz UA, Mohrmann S, Fischer J, et al. Comparison of rapidly cycled tandem high-dose chemotherapy plus peripheral-blood stem-cell support versus dose-dense conventional chemotherapy for adjuvant treatment of high-risk breast cancer: results of a multicentre phase III trial. Lancet. 2005;366:1935-44.

Park JR, Kreissman SG, London WB, et al. A phase III randomized clinical trial (RCT) of tandem myeloablative autologous stem cell transplant (ASCT) using peripheral blood stem cell (PBSC) as consolidation therapy for high-risk neuroblastoma (HR-NB): a Children's Oncology Group (COG) study. J Clin Oncol. 2016;34(18_Suppl):LBA3. https://doi. org/10.1200/JCO.2016.34.18_suppl.LBA3.

Passweg JR, Baldomero H, Bader P, et al. Is the use of unrelated donor transplantation leveling off in Europe? The 2016 European Society for Blood and Marrow Transplant activity survey report. Bone Marrow Transplant. 2018. https://doi.org/10.1038/ s41409-018-0153-1.

Pedrazzoli P, Martino M, Delfanti S, et al. High-dose chemotherapy with autologous hematopoietic stem cell transplantation in high-risk breast cancer patients. J Natl Cancer Inst. 2015;51:70-5.

Peinemann F, Labeit AM. Autologous haematopoietic stem cell transplantation following high-dose chemotherapy for non-rhabdomyosarcoma soft tissue sarcomas: a Cochrane systematic review. BMJ Open. 2014;4:e005033.
Presson A, Moore TB, Kempert P. Efficacy of high-dose chemotherapy and autologous stem cell transplant for recurrent Wilms' tumor: a meta-analysis. J Pediatr Hematol Oncol. 2010;32:454-61.

Raleigh DR, Tomlin B, Buono BD, et al. Survival after chemotherapy and stem cell transplant followed by delayed craniospinal irradiation is comparable to upfront craniospinal irradiation in pediatric embryonal brain tumor patients. J Neuro-Oncol. 2017;131:359-68.

Sauerbrey A, Bielack S, Kempf-Bielack B, et al. Highdose chemotherapy (HDC) and autologous hematopoietic stem cell transplantation (ASCT) as salvage therapy for relapsed osteosarcoma. Bone Marrow Transplant. 2001;27:933-7.

Sureda A, Bader P, Cesaro S, et al. Indications for allo and auto-SCT for haematological diseases, solid tumors, and immune disorders: current practice in Europe 2015. Bone Marrow Transplant. 2015;50:1037-56.

Tenneti P, Zahid U, Sagar F, et al. Role of high-dose chemotherapy and autologous stem cell transplantation for relapsed Ewing's sarcoma: a case report with focused review of literature. Cureus. 2018;10:e2581.

Thiel U, Wawer A, Wolf P, et al. No improvement of survival with reduced- versus high-intensity conditioning for allogeneic stem cell transplants in Ewing tumor patients. Ann Oncol. 2011;22:1614-21.

Thiel U, Koscielniak E, Blaeschke F, et al. Allogeneic stem cell transplantation for patients with advanced rhabdomyosarcoma: a retrospective assessment. $\mathrm{Br}$ J Cancer. 2013;109:2523-32.

Whelan J, Le Deley MC, Dirksen U, Euro-E.W.I.N.G.99 and EWING-2008 Investigators, et al. High-dose chemotherapy and blood autologous stem-cell rescue compared with standard chemotherapy in localized high-risk ewing sarcoma: results of EuroE.W.I.N.G.99 and Ewing-2008. J Clin Oncol. 2018. https://doi.org/10.1200/JCO.2018.78.2516

Yu AL, Gilman AL, Ozkaynak MF, et al. Anti-GD2 antibody with GM-CSF, interleukin-2, and isotretinoin for neuroblastoma. N Engl J Med. 2010;63:1324-34.

Open Access This chapter is licensed under the terms of the Creative Commons Attribution 4.0 International License (http://creativecommons.org/licenses/by/4.0/), which permits use, sharing, adaptation, distribution and reproduction in any medium or format, as long as you give appropriate credit to the original author(s) and the source, provide a link to the Creative Commons license and indicate if changes were made.

The images or other third party material in this chapter are included in the chapter's Creative Commons license, unless indicated otherwise in a credit line to the material. If material is not included in the chapter's Creative Commons license and your intended use is not permitted by statutory regulation or exceeds the permitted use, you will need to obtain permission directly from the copyright holder.

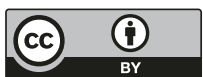

